\title{
Wet granulation in laboratory-scale high shear mixers: Effect of chopper presence, design and impeller speed
}

\author{
T.M. Chitu ${ }^{\mathrm{a}, *}$, D. Oulahna ${ }^{\text {a }}$, M. Hemati ${ }^{\mathrm{b}}$ \\ ${ }^{a}$ RAPSODEE Research Centre, UMR EMAC-CNRS 2392, Ecole des Mines d'Albi-Carmaux, Campus Jarlard, 81013 Albi, France \\ b Laboratoires de Génie Chimique UMR-CNRS 5503, ENSIACET, INPT, BP 1301, 5 Rue Paulin Talabot, 31106 Toulouse Cedex 1, France
}

Corresponding author at Rapsodee (oulahna@mines-albi.fr)

\begin{abstract}
A B S T R A C T
The effect of the main means of agitation in a high shear mixer has been investigated in this study. Granulation runs have been performed on a fine cohesive microcrystalline cellulose powder (Avicel 105, $\mathrm{d}_{50}=20 \mu \mathrm{m}$ ) often used as a pharmaceutical excipient in tablet formulations in two bowls of a Mi-Pro® laboratory high shear mixer with a capacity of 0.9 and $1.9 \mathrm{~L}$, respectively. Torque curves recorded during granulation are found to allow good control of the process while increasing impeller speed is found to generally reduce granule size and the onset of breakage seems to occur for similar values of impeller tip speed. As a general rule, the chopper allows for better binder distribution in the Mi-Pro® and is found to be necessary for successful granulation at low to moderate impeller speeds. For high impeller speeds in excess of $4.4 \mathrm{~m} / \mathrm{s}$ with or without a chopper, similar granule sizes and growth mechanisms are observed. Granule roundness was found to increase with impeller speed up to a certain speed after which granule roundness has been found to decrease with increasing impeller speed most probably because of increased breakage of the granules. Dry granule strength has been found to increase with increasing impeller speed, presenting only a slight decrease at the highest impeller speed studied.
\end{abstract}

\section{Introduction}

Wet granulation using high shear mixers is a widespread technique applied in fields such as pharmaceutical development and various branches of the chemical industry. Formulations are usually developed at small scale and following validation are scaled-up to production units. Most equipment manufacturers offer small-scale versions of their industrial equipment in order to allow a smoother transition.

The Mi-Pro high shear granulator allows working at different scales, keeping similar ratios between bowl, impeller and chopper and can be seen as a scaled-down Collette-Graal high shear mixer. The instrumentation of the Mi-Pro allows the recording of torque curves during granulation. As shown by Mackaplow et al. [1], some difficulties exist related to using torque/power curves as a means to control granulation given by their sensitivity to wall build-up and collapse; however, the relationship between torque/power curves and tensile strength discussed by Betz et al. [2] shows the promising future of this technique, which can relate material properties to operating conditions.

The effect of the chopper depends largely on the equipment used, while some authors like Michaels et al. [3] find no effect of the chopper on the granule size distribution, others have found that using the chopper slightly decreases the mean granule size, Schaefer et al. [4] or narrows the granule size distribution, Knight et al. [5]. Increasing the impeller speed generally leads to a decrease in granule size and an increase in growth rate. As scale-up techniques usually imply finding the equivalence of impeller speeds between the scales following certain rules, reviewed by Faure et al. [6], it seemed pertinent to investigate the effect of impeller speed on the Mi-Pro high shear mixer.

In situ monitoring and identification of granule growth kinetics are important aspects of the wet granulation process and transition between growth regimes can be influenced by changes in operating conditions as shown by Saleh et al. [7] and Benali et al. [8]. Describing high shear mixer granulation is considered especially difficult as agglomeration (growth), breakage and attrition are often simultaneous. Breakage and attrition depend on the mechanical strength of the wet agglomerate and the impact and shear forces developed by the main means of agitation present in the mixer. It is reasonable to assume that both chopper and impeller affect the consolidation of the granules while the high tip speed of chopper also ensures breakage of lumps. The chosen powder for this study is a fine microcrystalline cellulose powder. The granulation of this type of powders has been shown to go through a typical four-regime granulation: wetting, nucleation, growth and overwetting by Benali et al. [8] while Bouwman et al. [9] have shown that granules in the growth stage present a high deformability upon impact and shear with pieces of the main granules breaking off and coalescing with other granules followed by a new rearrangement to a sphere. 
Table 1

Physicochemical characteristics of binder solutions, contact angle and microcrystalline cellulose Avicel 105.

\begin{tabular}{llll}
\hline Binder properties & $\rho_{\mathrm{L}}\left(\mathrm{kg} / \mathrm{m}^{3}\right)$ & $\gamma_{\mathrm{L}}(\mathrm{mN} / \mathrm{m})$ & $\mu_{\mathrm{L}}\left(\mathrm{mPa} / \mathrm{s}\right.$ at $\left.25^{\circ} \mathrm{C}\right)$ \\
\hline Water & 1000 & 72.2 & 1.0 \\
Powder properties & $D_{50}[\mu \mathrm{m}]($ volumetric $)$ & Span $\left(D_{90}-D_{10}\right) / D_{50}$ & $69.0 \pm 1.2$ \\
\hline Avicel PH 105 & 20 & 1.93 & Bulk density $\left[\mathrm{kg} / \mathrm{m}^{3}\right]$ \\
Avicel PH 101 & 60 & 2.1 & 244 \\
\hline
\end{tabular}

Torque curves have been related to different states of liquid saturation [10]: pendular state when particles are held together by a liquid bridge at their contact points, capillary state that occurs when all the voids are filled with the liquid and the surface liquid is drawn back into the pores under capillary action and funicular state as a transition state between the pendular and capillary state where the voids are not fully saturated with liquid. Torque profiles allow the definition of these states as a function of the added binder where phase boundaries are marked at the intersection of drawn tangents to the curve with the optimum liquid amount supposed to be found in the plateau phase (growth zone) [11].

The aim of this study is to investigate the influence on the process of the main means of agitation in the high shear granulator (chopper and impeller) and the ability of torque curves to control it.

\section{Materials and Methods}

\subsection{Materials}

Microcrystalline cellulose Avicel PH 105 (FMC Biopolymer, Ireland) with a mean diameter of about $20 \mu \mathrm{m}$ has been chosen as a model product for this study. For comparison purposes, another microcrystalline cellulose grade, Avicel PH 101, with a mean diameter of $60 \mu \mathrm{m}$ has also been used. Some relevant properties for these materials are presented in Table 1 with ultra-pure water being used as a binder in all experiments.

\subsection{Granulation equipment: the Mi-Pro® high shear mixer}

The Mi-Pro (Pro-C-Ept, Zelzate, Belgium) high shear mixer allows granulating while recording real-time impeller torque values with 1 second intervals. This vertical axis high shear granulator can be equipped with differently sized glass bowls with corresponding threebladed impellers and choppers. Granulation time was $\sim 14$ minutes, the powder bed was homogenized by dry mixing for 2 minutes before the water addition was done at a constant rate using a Dosimat 760 syringe pump (Metrohm, Berchem, Belgium) through a 400- $\mu \mathrm{m}$ diameter capillary tube. Granulation was studied at two scales in the 0.9 and $1.9 \mathrm{~L}$ bowls (Fig. 1). Impellers have a similar design while the chopper is slightly inclined in the $0.9 \mathrm{~L}$ bowl.
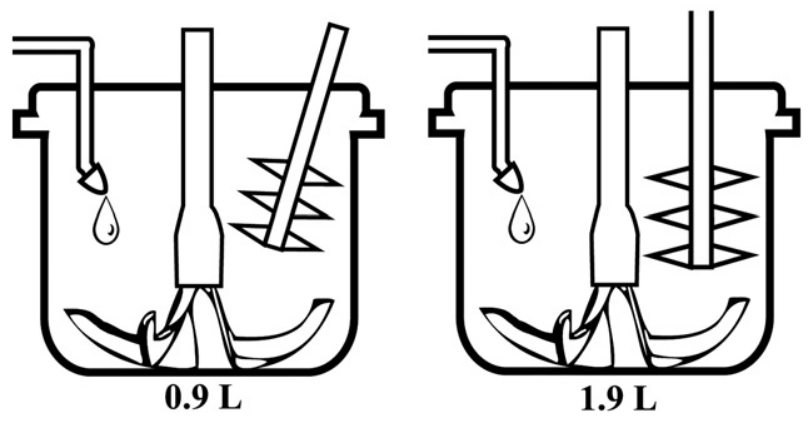

Fig. 1. Schematic representation of the investigated Mi-Pro bowls.
In order to assess granule growth and morphological properties, representative samples of solid (15-30 g) were removed from the bed and tray-dried at $40{ }^{\circ} \mathrm{C}$ overnight. Due to the small size of the mixer bowls and small mass of product handled (120 g MCC Avicel 105 for the $1.9 \mathrm{~L}$ bowl), each sample was taken from a new batch with experiments being carried out twice. The dried granules were sieved and 15 size fractions were collected. Weight mean diameter was determined using the following equation:

Mean diameter $: d_{p m}=\frac{\sum_{i} f_{i} d_{p i}}{\sum_{i} f_{i}}$

where $f_{i}$ is the particle mass fraction of size interval $i, d_{p i}$ is the mean diameter of size interval $i(\mu \mathrm{m})$. For a better understanding of agglomeration mechanisms, three characteristic granule classes were defined (Fig. 2): fine (corresponding to the initial powder with granule diameters and dry agglomerates inferior to $200 \mu \mathrm{m}$ ), intermediate (weak granules with diameters between 200 and $800 \mu \mathrm{m}$ ) and coarse agglomerate (strong, dense granules with diameters greater than $800 \mu \mathrm{m}$ but smaller than $5 \mathrm{~mm}$ ). Granules exceeding $5 \mathrm{~mm}$ are defined as lumps and are not taken into account in the mean diameter equation. Fig. 2 shows the defined classes at $50 \%$ liquid to solid ratio for an impeller speed of $800 \mathrm{rpm}$ and chopper speed of $3000 \mathrm{rpm}$. This definition is considered to allow a good description over a wide range of operating conditions; however, instances may occur where the mean diameter value and granulation kinetics analysis can be influenced by these imposed boundaries. For operating conditions leading to large agglomerates like low impeller and chopper speeds for instance where a low number of high diameter granules could be obtained or on the other hand for very high impeller speeds where granules would not grow above $800 \mu \mathrm{m}$ because of extended granule breakage leading to no coarse agglomerates class.

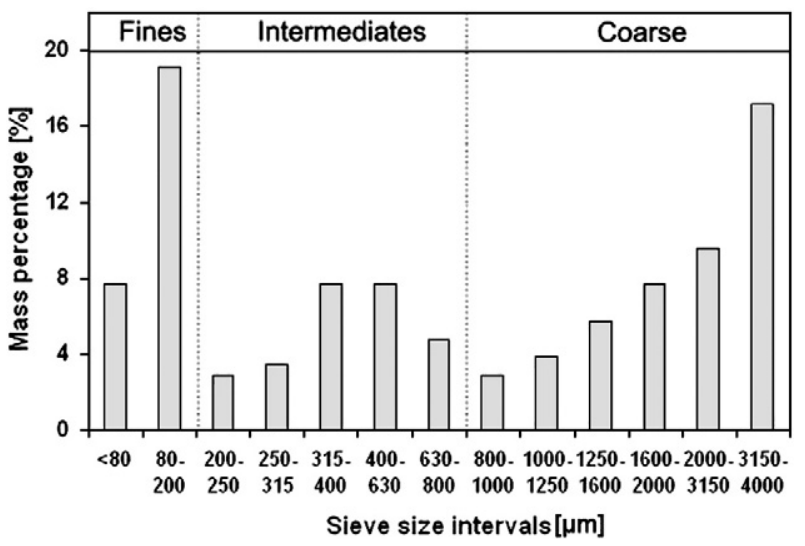

Fig. 2. Sieving results for a $\mathrm{L} / \mathrm{S}$ ratio of $50 \%$ at an impeller speed of $800 \mathrm{rpm}$ and a chopper speed of $3000 \mathrm{rpm}$ for the granulation of MCC Avicel 105 with water. 


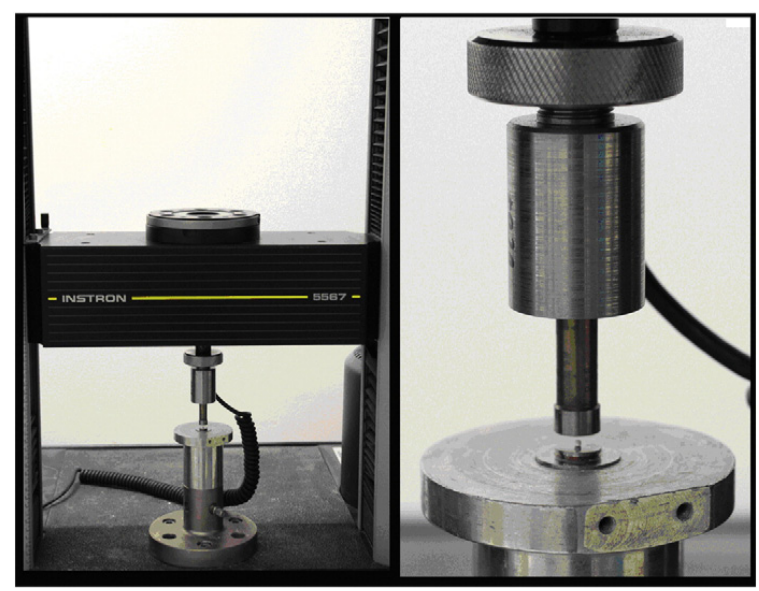

Fig. 3. The Instron 5567 mechanical testing machine.

Kinetic results are expressed in function of the fraction added liquid to initial dry powder mass named liquid to solid ratio:

L / S Ratio = Added Liquid Mass [kg] / Initial Powder Mass [kg] (\%) (2)

Discussing kinetics as a function of added liquid will always present a certain degree of relativity as the added liquid may or may not correspond to the granule saturation, especially when poor liquid distribution is involved. However, with products like microcrystalline cellulose where liquid-solid interaction involves swelling [12] and formation of a crystallite gel [13] during pelletisation, calculating saturation is extremely difficult.

When discussing the effects at a single scale, impeller speeds have been expressed in revolutions per minute; when comparing scales or when expressing behavior common to multiple scales, the impeller tip speed has been chosen as the common denominator.

\subsection{Granule strength and roundness}

Granule strength was evaluated by single granule compression tests using an Instron 5567 mechanical testing machine (Fig. 3). The Instron testing machine allows recording a maximum force of $500 \mathrm{~N}$, sufficient to break all of the tested granules. When the granule is subjected to compressive strain (displacement), a marked drop in the signal can be observed when the granule first cracks (Fig. 4). The values recorded after this point on the load-displacement curve are of little practical use as they represent the compression of granule fragments and eventually powder particles. Granules tested had a diameter close to the mean a)

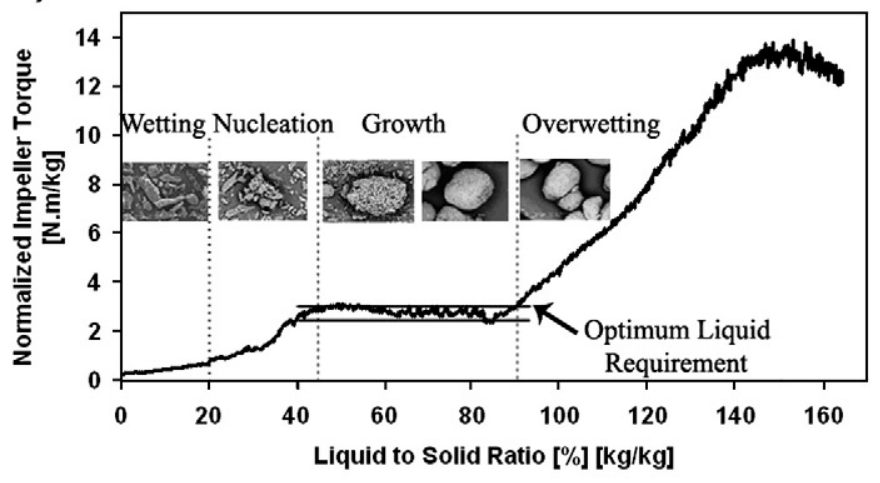

b)

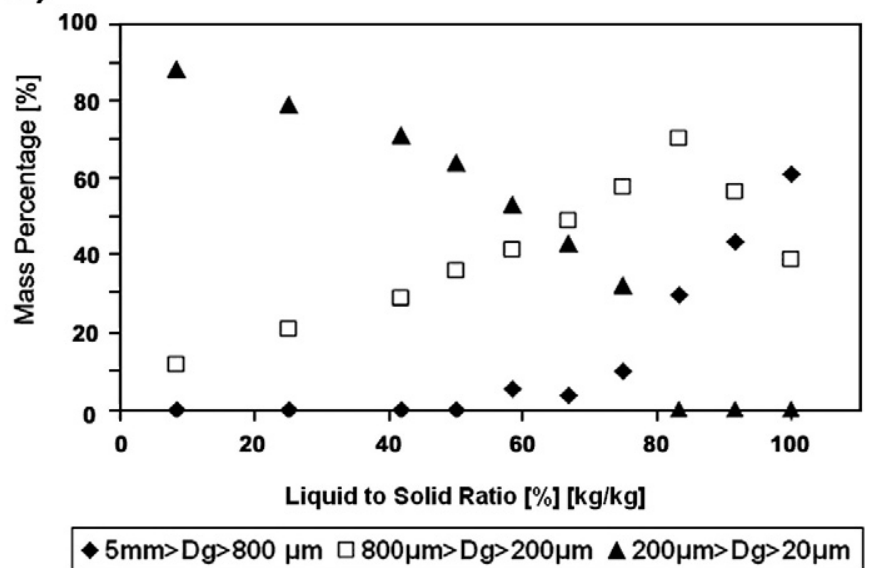

Fig. 5. Torque curve (a) and characteristic fraction evolutions (b) obtained on the $1.9 \mathrm{~L}$ Mi-Pro HSM for the granulation of MCC Avicel 105 with water.

diameter of the granulation run. Granule strength was assessed using the following equation proposed by Adams et al. [14]:

$\sigma_{g}=4 \frac{F_{\max }}{\pi \cdot D_{g}^{2}}$

Where $F_{\max }$ is the fracture force and $D_{\mathrm{g}}$ is the measured granule diameter.

Imposed velocity of the steel probe was of $1 \mathrm{~mm} / \mathrm{min}$ while the tested granules diameter was in the 1000 to $1250 \mu \mathrm{m}$ range.

Granule roundness was determined by an automated microscopy and image analysis technique (PharmaVision 830, Malvern Instruments, Inc., USA). Roundness is a measurement relating length to width and
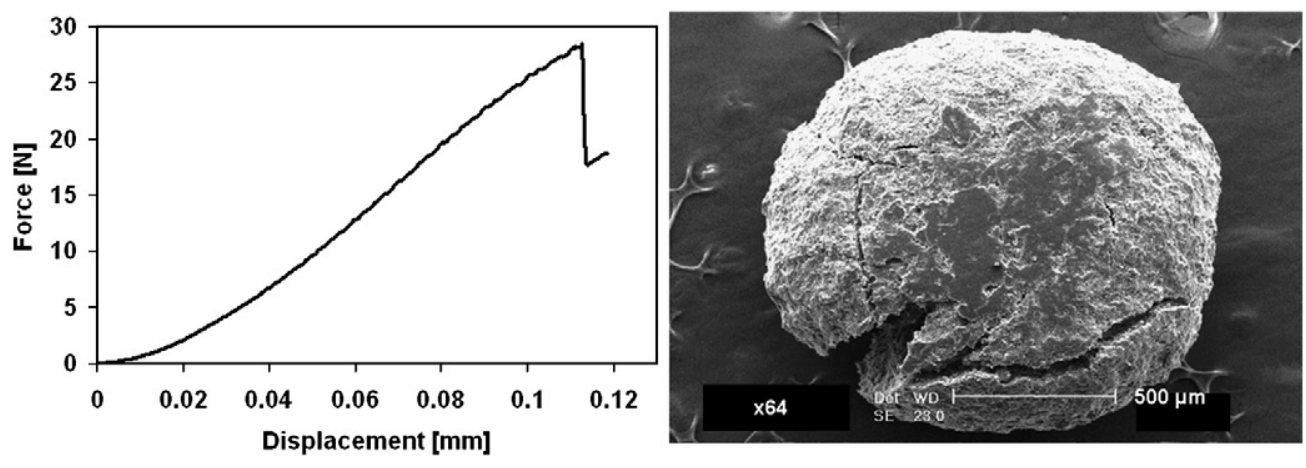

Fig. 4. Characteristic load displacement curve and scanning electron microscope picture of the cracked granule. 
a)

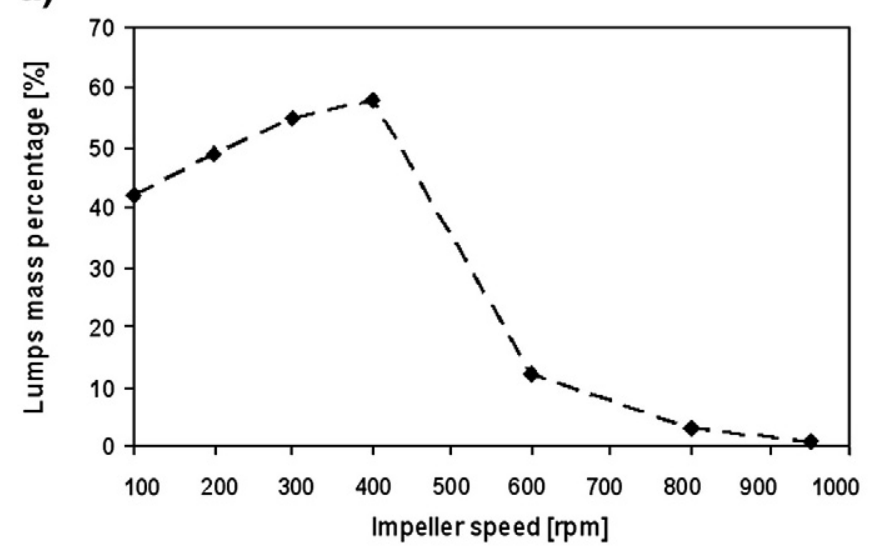

b)

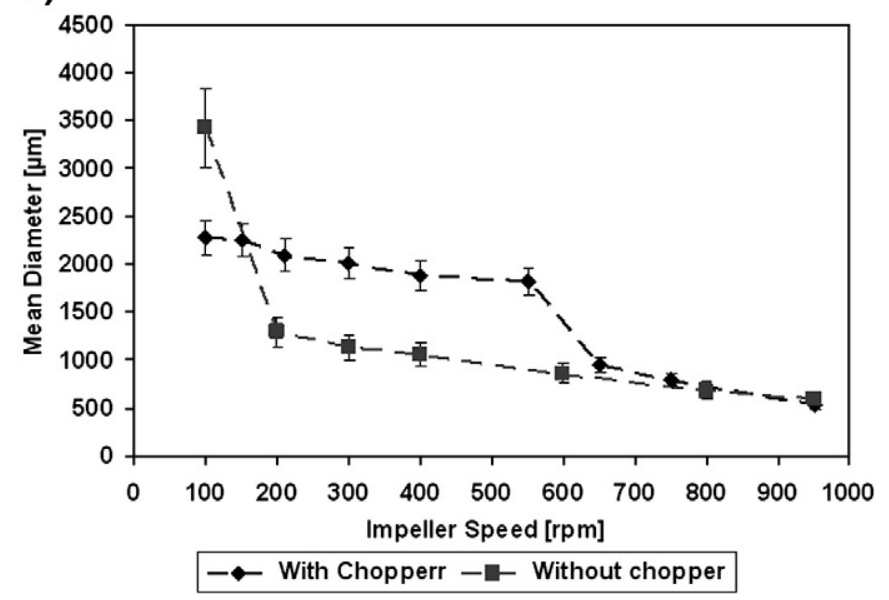

Fig. 6. Evolution of the lump fraction (granules with diameter above $5 \mathrm{~mm}$ ) as a function of impeller speed (a) and mean granule size evolution as a function of impeller speed (b) in the Mi-Pro HSM V =1.9 L for the granulation of MCC Avicel 105 with water.

presenting values in the range of 0 to 1 . A perfect circle would present a roundness of 1 while a needle-shaped particle would have a roundness close to 0 . The roundness is determined typically using the equation:

Roundness $=\frac{4 \pi A_{g}}{P_{g}^{2}}$

where $A_{\mathrm{g}}$ is the measured area and $P_{\mathrm{g}}$ is the perimeter of the granules. The zoom objective was used at the 0.5 position at which it presents a resolution of $18 \mu \mathrm{m}$, a depth of field of $11 \mu \mathrm{m}$, a field of view of $4722 \times 6166 \mu \mathrm{m}$ and can analyze granules in the size range of 45 to
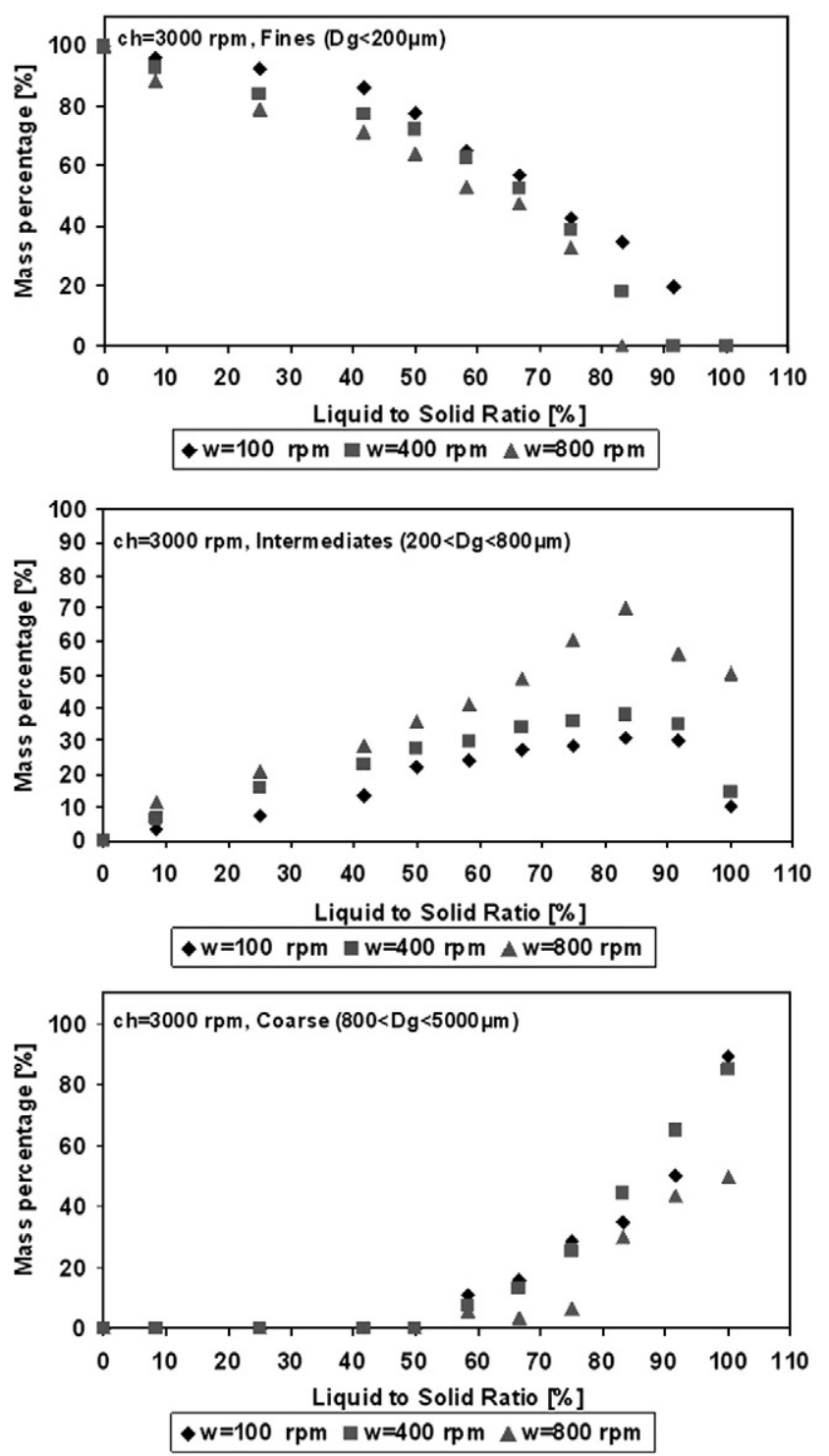

Fig. 8. Evolution of the characteristic size fractions with chopper rotating at $3000 \mathrm{rpm}$ for three impeller speeds in the Mi-Pro HSM V $=1.9 \mathrm{~L}$ for the granulation of MCC Avicel 105 with water.

$1908 \mu \mathrm{m}$. Granules investigated for roundness were in the range of 1000 to $1250 \mu \mathrm{m}$. Results can be expressed as a function of number or value distributions; however, in our experiments, both methods presented very similar values as well as nearly identical trends.
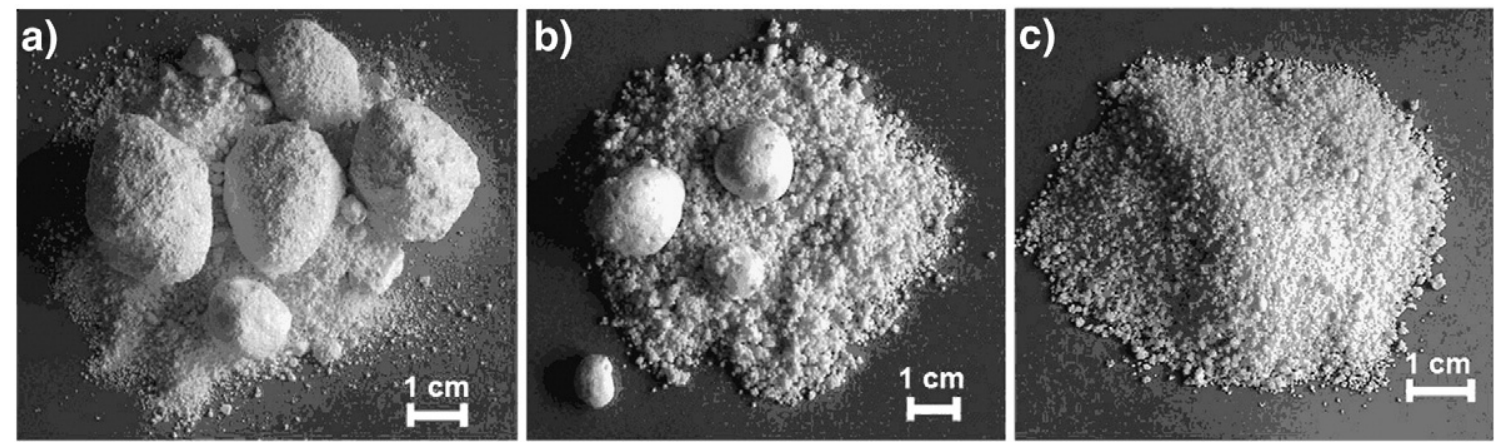

Fig. 7. Evolution of the final granulated product in the Mi-Pro HSM V=0.9 L without a chopper for three different speeds: (a) $300 \mathrm{rpm}$, (b) $650 \mathrm{rpm}$ and (c) $950 \mathrm{rpm}$ for the granulation of MCC Avicel 105 with water. 


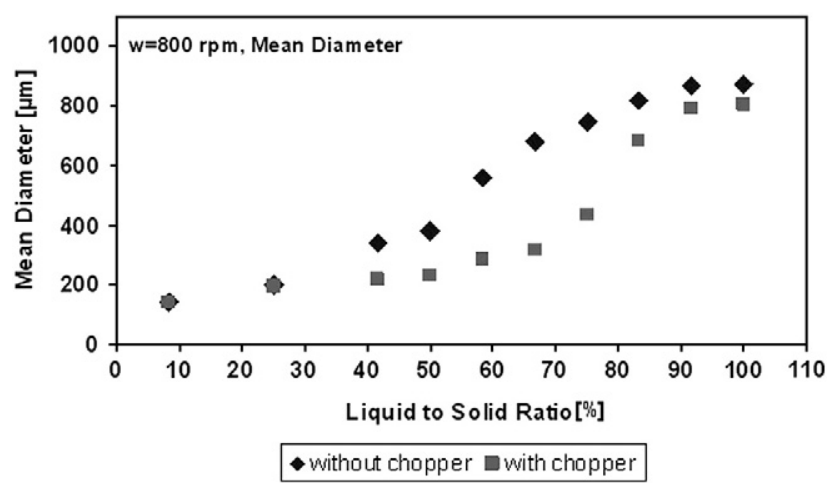

Fig. 9. Mean granule size evolution on the Mi-Pro $V=1.9 \mathrm{~L}$ with and without chopper at $800 \mathrm{rpm}$ for the granulation of MCC Avicel 105 with water.

\section{Results and discussion}

\subsection{Wet granulation of MCC Avicel 105 on the Mi-Pro HSM}

Fig. 5a shows scanning electron microscope (SEM) pictures of granules obtained from microcrystalline cellulose and water on the Mi-Pro high shear mixer as a function of liquid to solid ratio and the corresponding torque curve. Fig. 5b shows the evolution of the three characteristic classes, for an impeller speed of $800 \mathrm{rpm}$ and a chopper speed of $3000 \mathrm{rpm}$ allowing us to determine the optimum $\mathrm{L} / \mathrm{S}$ ratio for the water-MCC Avicel 105 granulation:

- for values of the S/L ratio between $0 \%$ and $20 \%$, wetting occurs with only a very small percentage of fines forming into intermediates,
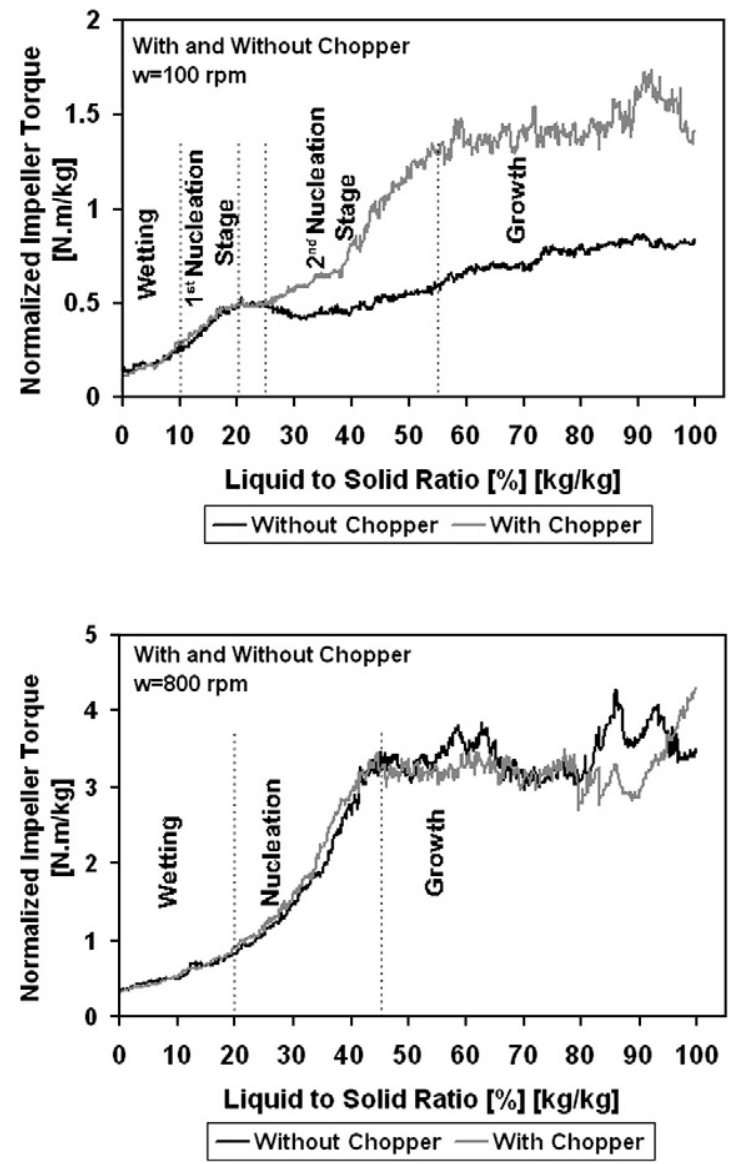

most probably because of the very cohesive nature of the initial powder rather than binder addition

- for values ranging from $20 \%$ to $~ 45 \%$, nucleation occurs with fines forming intermediate agglomerates in the 200 to $800 \mu \mathrm{m}$ range

- for $\mathrm{L} / \mathrm{S}$ ratios exceeding $50 \%$, growth occurs by layering and coalescence, first with the fine particles being agglomerated into intermediate granules which achieve a maximum value at 83\% L/S ratio and the apparition of the first coarse granules, secondly for values exceeding $83 \%$ with agglomeration between intermediate particles

- for $\mathrm{L} / \mathrm{S}$ ratios above $100 \%$, overwetting occurs, while some usable granules could still be obtained up to $125 \% \mathrm{~L} / \mathrm{S}$ ratio, the yield is low and presents a high percentage of lumps. For values above 125\%, L/S ratio the wet mass evolves to highly cohesive paste which upon further addition for $\mathrm{L} / \mathrm{S}$ ratios above $150 \%$ turns into a suspension.

While the torque curve is seperated into clear-cut segments as a function of its inflexion points (as defined by Leuenberger et al. [11], granule growth mechanisms transcend these limits and it is not uncommon to find nuclei for instance in the wetting stage as well as in the growth stage. However, the SEM observations allowed us to define the dominating mechanism for the presented $\mathrm{L} / \mathrm{S}$ ratio ranges.

\subsection{Effect of impeller speed and chopper presence and design}

The influence of chopper on mean granule size is discussed in Fig. 6. Without a chopper, between 100 and $400 \mathrm{rpm}$ lumps are found to increase with impeller speed (Fig. 6a), leading to an inhomogenous product comprised of small fine undergranulated product and a large percentage of lumps. This explains the high difference in mean
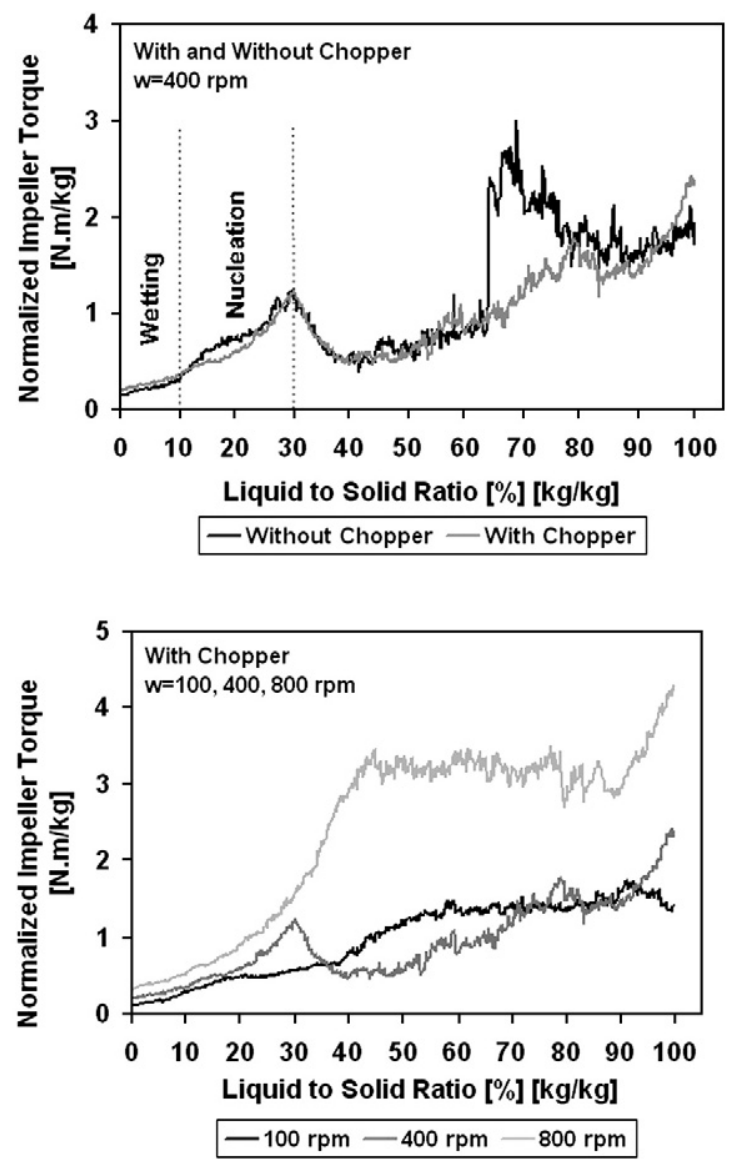

Fig. 10. Torque curve comparison for the Mi-Pro V = 1.9 L HSM with and without chopper at three different impeller speeds: 100,400 and 800 rpm and comparison between speeds with chopper active for the granulation of MCC Avicel 105 with water. 
granule size between 150 and $500 \mathrm{rpm}$ as lumps (granules above $5 \mathrm{~mm}$ ) are not taken into account when calculating the mean granule size (Fig. 6b). The single higher granule size value for granulation without a chopper against granulation with chopper is obtained at $100 \mathrm{rpm}$ and can be explained by our choice in defining lumps only for granules exceeding $5 \mathrm{~mm}$ as a mean granule size of $3.5 \mathrm{~mm}$ is still high and indicative of less than perfect granulating conditions. From $600 \mathrm{rpm}$ onwards, while a small percentage of lumps may still exist, the impeller speed alone becomes sufficiently high so as to break the lumps and reduce the percentage to a negligible value at $800 \mathrm{rpm}$ and beyond. It should also be noted that the high impeller speed also distributes the liquid binder better. Because the binder is added through a capillary and not sprayed, directing the liquid flow towards the rotating chopper also insures a better distribution of the binder over the powder bed. The same behavior showing that the lump fraction decreases with increasing impeller speed when granulating without a chopper has also been observed on the $0.9 \mathrm{~L}$ Mi-Pro granulation bowl. Fig. 7 shows pictures of representative samples taken from the granulator at three different speeds when granulating without a chopper, showing diminishing amounts of lumps with increasing impeller speed.

Setting the chopper at a high rotating speed and investigating the influence of impeller speed at 100, 400 and $800 \mathrm{rpm}$ on the growth kinetics, we can observe that increasing impeller speed accelerates the consumption of fine particles and the formation of intermediates and coarse granules (Fig. 8). While for the lower impeller speeds, a decrease in intermediate particle percentage is observed near the end of the granulation runs for $800 \mathrm{rpm}$; the intermediate particles show the highest percentage and are still present at the end of the granulation. This can be explained by the fact that at $800 \mathrm{rpm}$, breakage occurs and with a mean granule size of $800 \mu \mathrm{m}$, the intermediate fraction $200-800 \mu \mathrm{m}$ does not contain low-density, fragile granules, but rather high-density broken-down granules. When looking at what happens with or without a chopper at $800 \mathrm{rpm}$, where the chopper would not be necessary in regards to mean final granule size, we see however a significant difference in terms of granule growth, with the chopper delaying the formation of coarse granules (Fig. 9).

The effect of chopper presence on torque curves with the chopper turning at $3000 \mathrm{rpm}$ shows only small differences between operating with or without chopper at medium and high impeller speeds (Fig. 10). On the $400 \mathrm{rpm}$ curve after the wetting stage (up to10\% L/ $\mathrm{S}$ ratio) a sharp drop in torque can be observed at $30 \% \mathrm{~L} / \mathrm{S}$ ratio. Where torque curves are continuous (100 and $800 \mathrm{rpm}$ ), we observe an evolution similar to that proposed by Leuenberger [11]. Also at 400 and $800 \mathrm{rpm}$, the chopper influence is not visible, the recorded torque curve showing the same inflexion points independent of chopper use. We expected the differences in torque curves for varying impeller speeds to be relateable to granulation kinetics obtained by sieving of the dry product. It would seem however that our sieving protocol homogenizes the results between the studied impeller speeds being perhaps too destructive with the agglomerates obtained at low impeller speeds, which as we will show present a lower granule strength.

For the low impeller speed of $100 \mathrm{rpm}$, we observed that the chopper turning at high speed can favor granulation. Granulation at $100 \mathrm{rpm}$ with the chopper active is characterised by an initial wetting stage up to $10 \% \mathrm{~L} / \mathrm{S}$ ratio followed by a first stage of nucleation. However, while we use the same name for granulation mechanisms, it should be stated that at $100 \mathrm{rpm}$, the nuclei formed in the first nucleation stage are few but big, low-resistance agglomerates resulting mainly from layering. This nucleation mechanism is a result of poor binder dispersion at such low impeller speeds and is not similar to the nucleation observed at 400 and $800 \mathrm{rpm}$. Once the agglomerates are large enough, they come in contact with the chopper and a redistribution of moisture and material occurs. We call the resulting re-agglomeration as a secondary nucleation stage because of the similar increase in torque signal with the nucleation stage. Denser, smaller nuclei were observed to result from the brokenup larger agglomerates. For the granulation run without chopper at $100 \mathrm{rpm}$, the second nucleation stage and redistribution of powder and binder does not take place and the end result corresponds to granules presenting a much larger mean granule size.

At $400 \mathrm{rpm}$, the drop in torque is characteristic for the granulation of MCC Avicel 105 at moderate speeds in the Mi-Pro and is independent of chopper operation: when sufficient liquid is added, the powder sticks to the side walls exiting the impeller action causing a sharp drop in impeller torque. This seems to be related to the mechanical dispersion of the binder in the powder bed as well as the small particle size and high compressibility of the MCC Avicel 105. Fig. 11 shows a torque comparison between MCC Avicel 105 $\left(D_{50}=20 \mu \mathrm{m}\right)$ and MCC Avicel $101\left(D_{50}=60 \mu \mathrm{m}\right)$ and the reproducibilty for MCCAvicel 101.

To better illustrate the difference in behavior between these two powders at $400 \mathrm{rpm}$, we performed a granulation run using water coloured with a red tracer. Fig. 12 shows the contents of the bowl for MCC Avicel 105 and Avicel 101. Fig. 13 shows the bowl after the product has been emptied from the bowl. As it can be observed in Fig. 12a, there is a strong interaction between the MCC Avicel 105 powder and the glass walls of the Mi-Pro while the same cannot be observed for the MCC Avicel 101 where the product stays inside the bowl without sticking to the walls. When emptying the bowls, we can observe that for both powders, a certain amount of powder is being compressed to the glass wall in the space between impeller and wall. The red stains formed
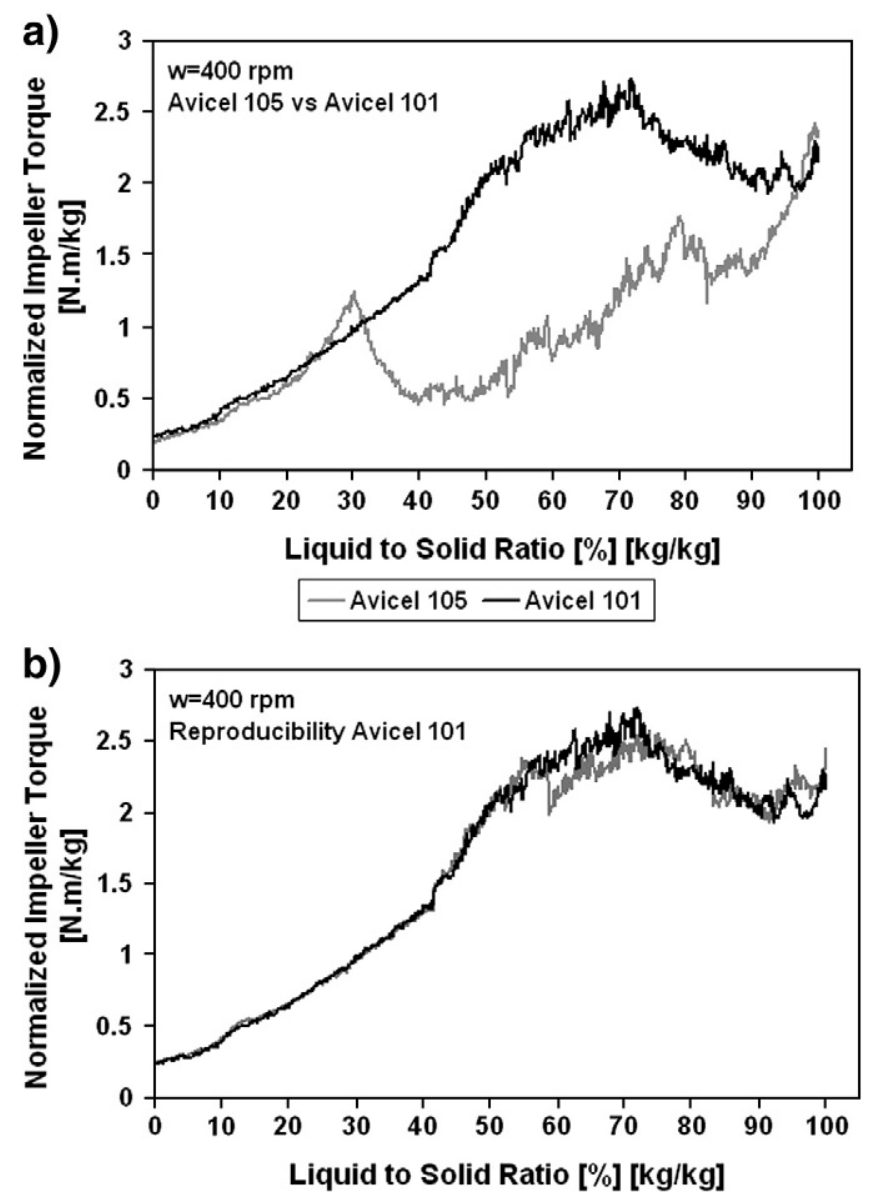

Fig. 11. Comparison between torque curves at $400 \mathrm{rpm}$ for the Mi-Pro V=1.9 L HSM with chopper active for MCC Avicel 101 and 105 (a) and reproducibility for MCC Avicel 101 (b). 
a) Avicel 105

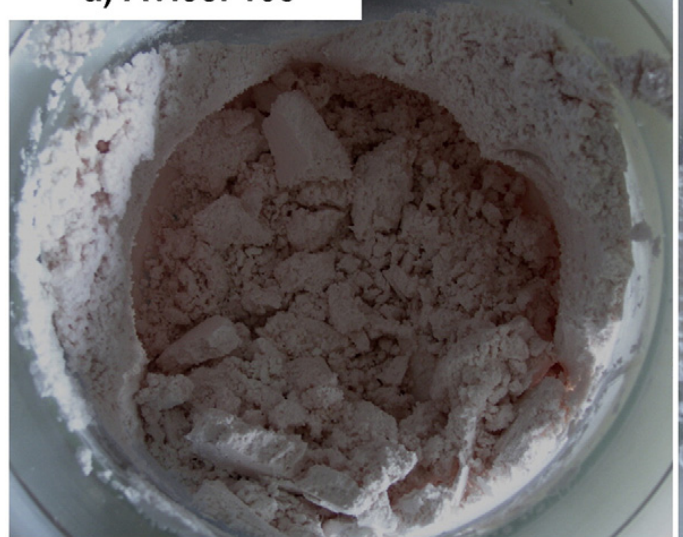

b) Avicel 101

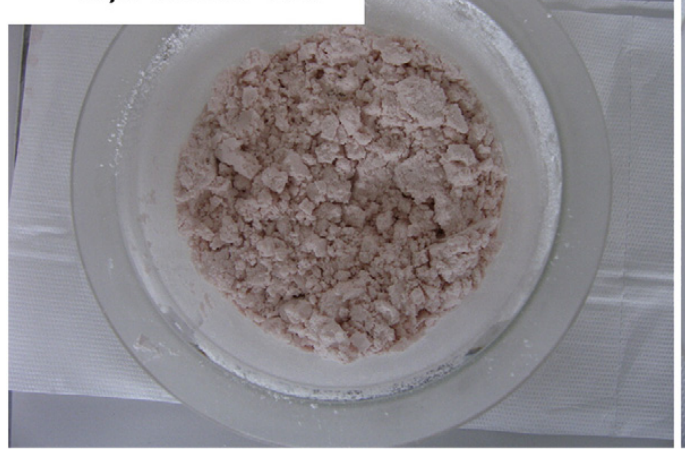

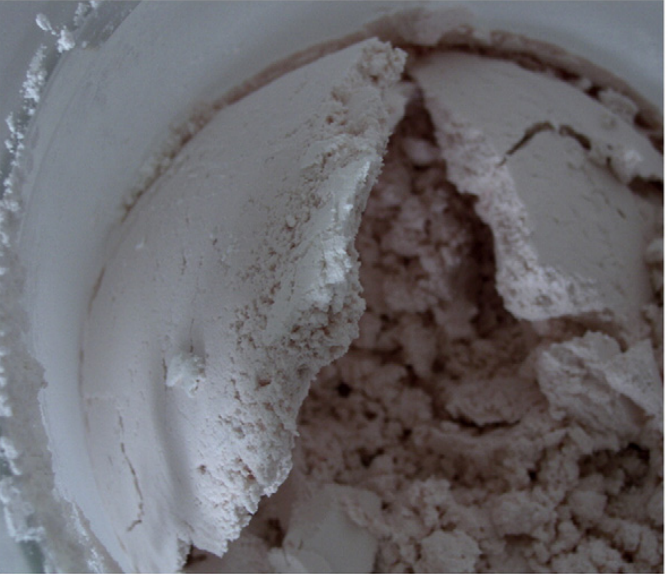

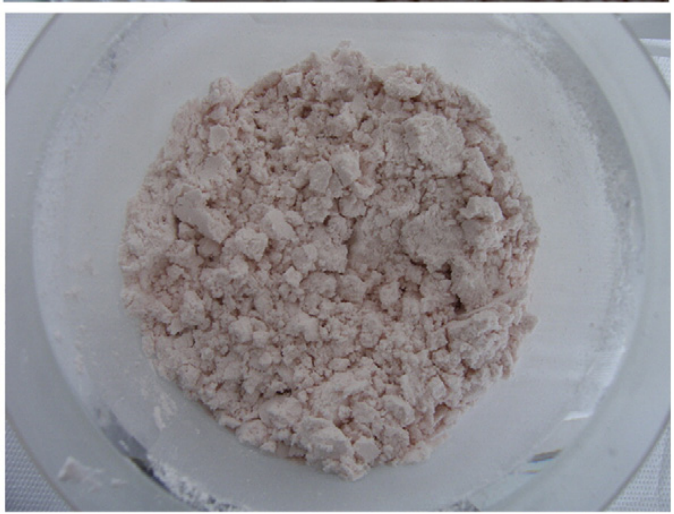

Fig. 12. View from above of the full Mi-Pro 1.9 L HSM at $400 \mathrm{rpm}$ with chopper active at 41\% L/S ratio for the granulation of MCC Avicel 105 (a) and Avicel 101 (b).

where the coloured water has been added show a much greater local overwetting for MCC Avicel 105 than for Avicel 101.

As noted by Hapgood et al. [15] for different grades of lactose, penetration times can vary greatly with mean particle size. They varied the mean surface diameter between 26 and $69 \mu \mathrm{m}$ and mean weight diameters between 67 and $200 \mu \mathrm{m}$ (roughly three times as is the case for the difference between MCC Avicel 105 and Avicel 101). This phenomenon could account for the different behavior in the nucleation phase.

Hapgood et al. [16] proposed a nucleation regime map describing a dimensionless penetration time parameter as a function of a dimensionless spray flux.

$\tau_{h}=\frac{t_{p}}{t_{c}}$

$\Psi_{a}=\frac{3 V^{\prime}}{2 A^{\prime} d_{d}}$

where $t_{\mathrm{p}}$ is the penetration time and $t_{\mathrm{c}}$ the circulation time of the powder in the nucleation zone, $V^{\prime}$ is the volumetric spray rate, $A^{\prime}$ the area flux of powder traversing the spray zone and $d_{\mathrm{d}}$ the droplet diameter.

While the penetration time is largely a function of formulation properties, the adimensional spray flux $\psi_{\mathrm{a}}$ depends on the operating conditions. The regime describes three regimes of nucleation:

- the drop-controlled regime where each individual drop completely wets the powder bed to form a single nuclei granule and nuclei size distribution is controlled by the drop size distribution,

- shear-controlled regime (or mechanical dispersion regime) where liquid pooling or caking occurs where the binder meets the bed and binder distribution occurs only by breakage of lumps due to shear forces inside the powder bed,

- and an intermediate regime between droplet- and shear-controlled where some agglomeration does occur near the spray zone without complete caking or pooling.

The presumable difference in penetration time difference for our two MCC grades could explain taking the powder from the drop-controlled regime (or intermediate) to the mechanical dispersion controlled regime where good binder dispersion requires good mechanical mixing. Given the binder "footprint" in Fig. 13b, it is reasonable to assume that granulation for MCC Avicel 101 at $400 \mathrm{rpm}$ takes place in the intermediate regime while granulation for MCC Avicel 105 occurs in the shear-controlled regime. In our case, the same binder flow rate is imposed through the same binder addition method (same average drop size), which leaves the velocity of the powder surface as the only free variable in estimating $\psi_{\mathrm{a}}$. We could not give an estimation for this velocity as our high shear mixer lacked the instrumentation with a highspeed video camera and image analysis software or positron emission particle tracking technology, two of the methods currently employed in order to measure powder surface velocity. However, as shown by Hapgood et al. [16] for water pumped on a lactose bed, the values for $\psi_{\mathrm{a}}$ would be close to a value of unity (in a 25 L Fielder high shear mixer), which would make it borderline between the intermediate and the shear-controlled regime.

We compared the effect of chopper design (length) by mounting the smaller and shorter chopper that equips the $0.9 \mathrm{~L}$ bowl on the $1.9 \mathrm{~L}$ bowl in order to further study the importance of the chopper for the Mi-Pro high shear mixers. The impeller speed chosen was of $650 \mathrm{rpm}$, considered sufficient to bring the powder mass in contact with both 

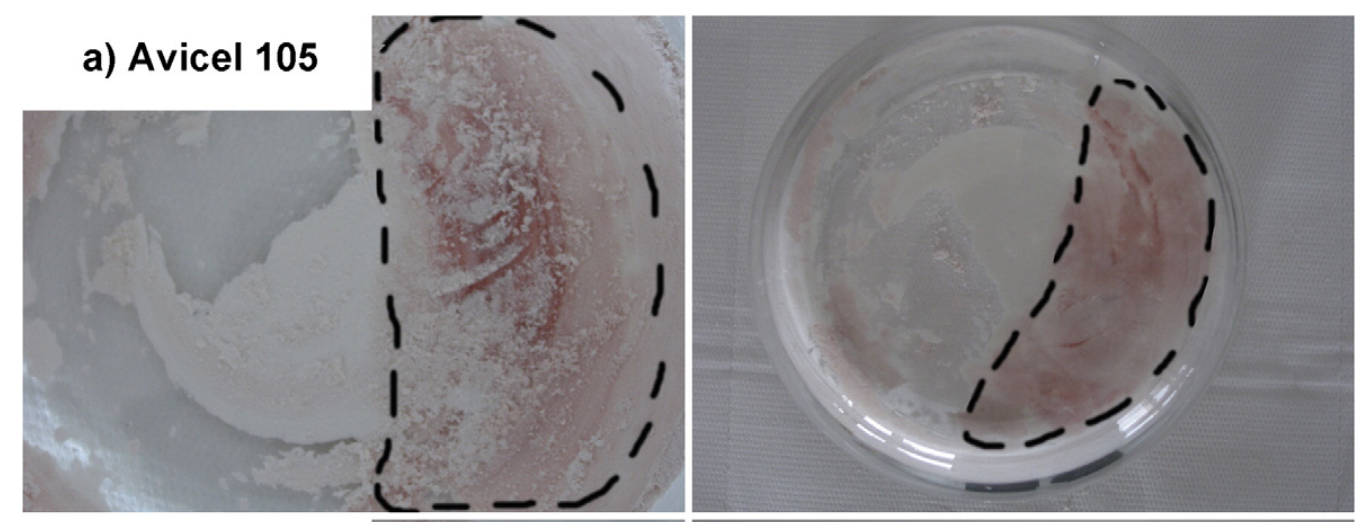

b) Avicel 101
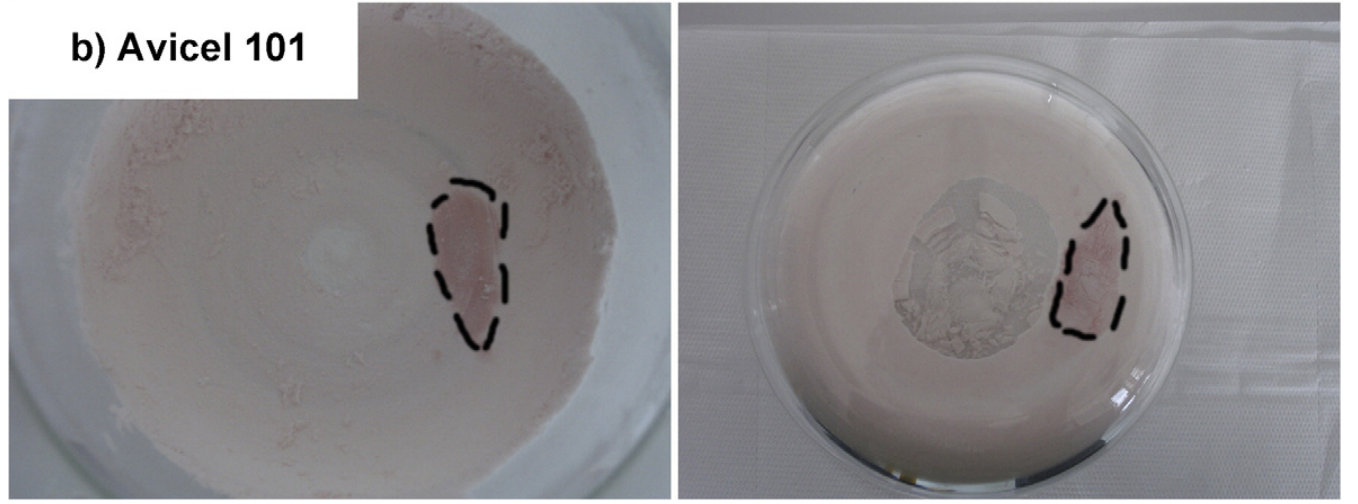

Fig. 13. Front and back view from above of the empty Mi-Pro $1.9 \mathrm{~L}$ bowls at $400 \mathrm{rpm}$ with chopper active at $41 \% \mathrm{~L} / \mathrm{S}$ ratio for the granulation of MCC Avicel 105 (a) and MCC Avicel 101 (b).

choppers. As can be observed in Fig. 14, there is a difference in granule size as well as amount of lumps. We obtained a mean granule size of $1200 \mu \mathrm{m}$ with the smaller chopper and $950 \mu \mathrm{m}$ with the regular one (20\% difference).

\subsection{Granule roundness and strength and mixer design influence}

Using the granules in the 1000 to $1250 \mu \mathrm{m}$ range, the roundness of the obtained granules was studied. Table 2 and Fig. 15 show the evolution of roundness with increasing impeller speed. Up to $400 \mathrm{rpm}$, the roundness increases while for values above $400 \mathrm{rpm}$, granules begin to decrease in roundness.
Dry granule strength measurements (Fig. 16) show that increasing the impeller speed leads to an increase in granule strength. Above $800 \mathrm{rpm}$, however, a slight decrease in granule strength was also observed which could be related to granule breakage becoming dominant to such an extent that granule growth and consolidation are affected while granule roundness decreases even more, making the use of Eq. (3) for granule strength, in the absence of a spheronization protocol at the end of granulation, questionable. It should also be mentioned that the granules obtained at $100 \mathrm{rpm}$ in the studied granule size range $(1000-1250 \mu \mathrm{m})$ show a lower reproducibility being very fragile.

As we have shown when discussing chopper influence, mean granule size depends largely on impeller speed, with sizes decreasing
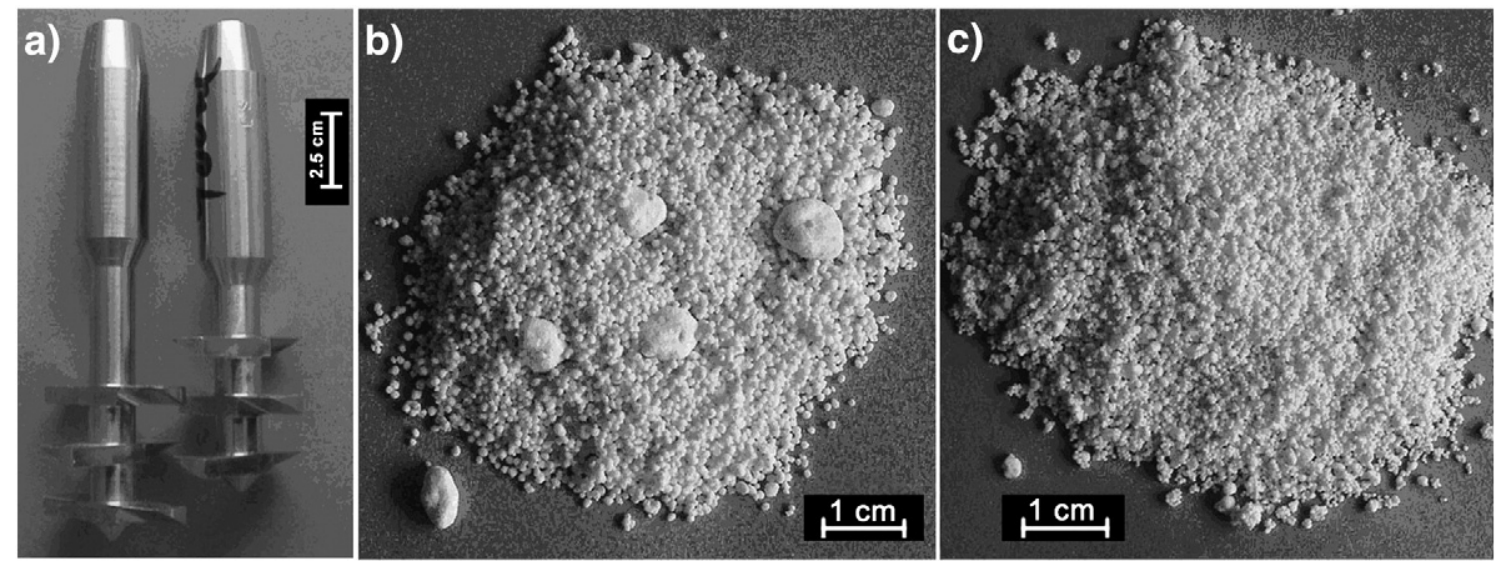

Fig. 14. (a) Choppers from the Mi-Pro HSM $1.9 \mathrm{~L}$ (left) and $0.9 \mathrm{~L}$ (right), (b) granules obtained in the $1.9 \mathrm{~L}$ bowl using the $0.9 \mathrm{~L}$ chopper, (c) granules obtained in the $1.9 \mathrm{~L}$ bowl using the 1.9 L chopper. 
Table 2

MCC Avicel 105 roundness as a function of impeller speed on the 1.9 L Mi-Pro bowl with the chopper rotating at $3000 \mathrm{rpm}$.

\begin{tabular}{lll}
\hline Impeller speed $[\mathrm{rpm}]$ & \multicolumn{2}{l}{ Roundness } \\
\cline { 2 - 3 } & $D_{50}$ & Span $=\left(D_{90}-D_{10}\right) / D_{50}$ \\
\hline 100 & 0.67 & 0.7 \\
260 & 0.77 & 0.43 \\
400 & 0.86 & 0.45 \\
550 & 0.79 & 0.47 \\
800 & 0.72 & 0.54 \\
1050 & 0.58 & 0.48 \\
\hline
\end{tabular}

upon increasing impeller speed. Comparing final granule size between two scales of the Mi-Pro: 0.9 and $1.9 \mathrm{~L}$ (Fig. 17) with similar geometric proportions in terms of bowl and impeller shape and expressing the impeller speed as impeller tip speed, we found a greater granule size for speeds lower than $1.5 \mathrm{~m} / \mathrm{s}$ on the small scale $(0.9 \mathrm{~L})$. This seems influenced by the same limit imposed for the definition of lumps where a higher degree of agglomerates below the $5 \mathrm{~mm}$ boundary can have a great impact on mean granule size on the smallest impeller speeds tested while even slightly larger granules than $5 \mathrm{~mm}$ are not taken into account. For speeds between 1.5 and $4.03 \mathrm{~m} / \mathrm{s}$, final granules are consistently larger on the larger scale $(1.9 \mathrm{~L})$; however, the difference does not seem dramatic. The evolution of slowly decreasing mean granule sizes is common to both scales, for values above $4.03 \mathrm{~m} / \mathrm{s}$, breakage occurs; and for values of $4.7 \mathrm{~m} / \mathrm{s}$ and above, granule sizes become identical. Our results are similar to those obtained by Ameye et al. [17] who have shown that the impeller tip speed does not have a

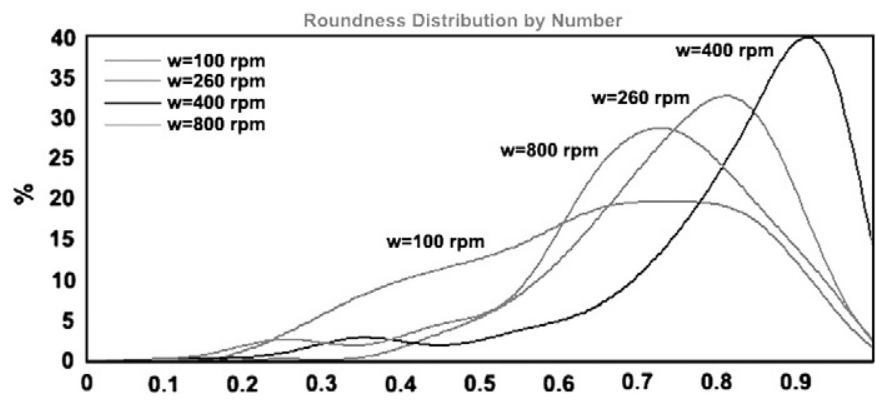

Fig. 15. Roundness distributions by number as a function of impeller speed for the granulation of MCC Avicel 105 with ultra-pure water on the $1.9 \mathrm{~L}$ Mi-Pro with the chopper at $3000 \mathrm{rpm}$.

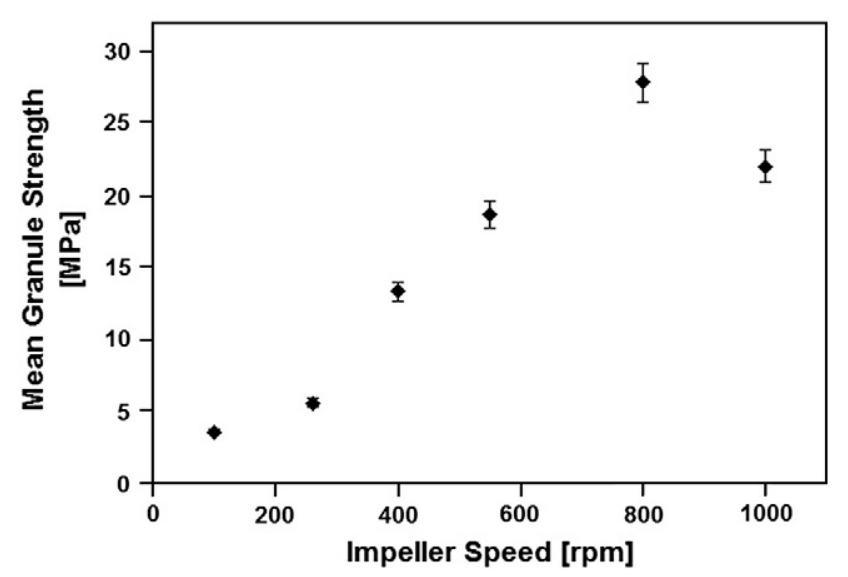

Fig. 16. Granule strength evolution as a function of impeller speed for the granulation of MCC Avicel 105 with ultra-pure water on the $1.9 \mathrm{~L}$ Mi-Pro with the chopper at $3000 \mathrm{rpm}$.

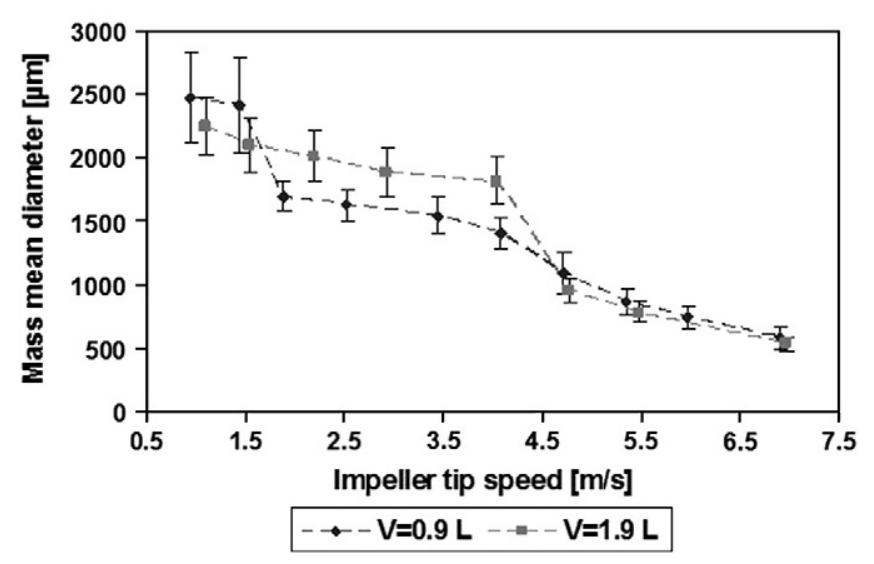

Fig. 17. Evolution of mean granule size as function of impeller tip speed on two scales of the Mi-Pro HSM: 0.9 L and 1.9 L for the granulation of MCC Avicel 105 with ultra-pure water.
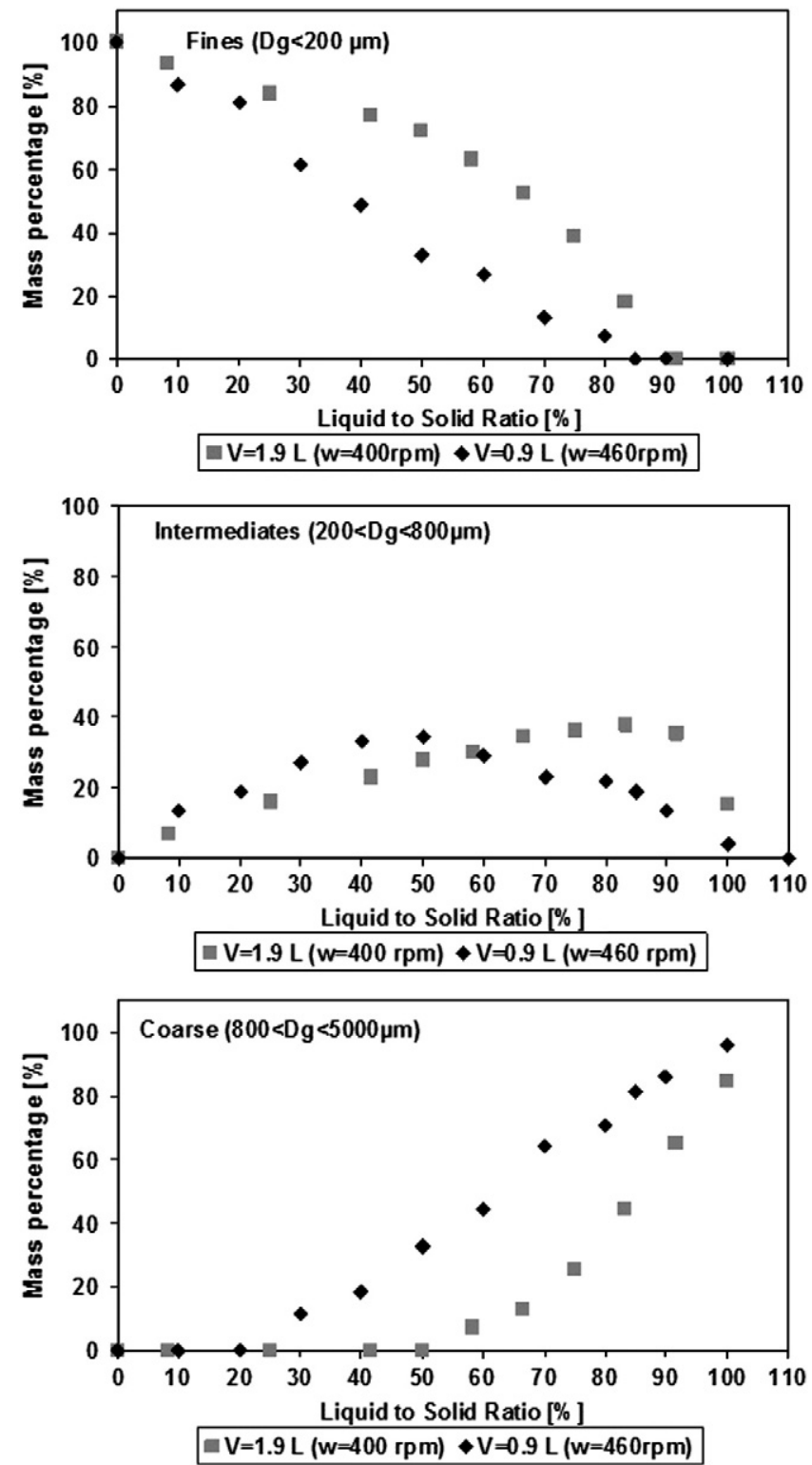

Fig. 18. Evolution of the characteristic size fractions for the same impeller tip speed in the Mi-Pro HSM V $=1.9 \mathrm{~L}$ and V $=0.9 \mathrm{~L}$ with the choppers at $3000 \mathrm{rpm}$ for the granulation of MCC Avicel 105 with ultra-pure water. 
dramatic effect on mean granule size between the 0.9 and 1.9 L bowls for impeller speeds above $5 \mathrm{~m} / \mathrm{s}$.

In order to investigate differences between scales, we chose to look in more detail at the growth mechanisms at nearly identical impeller tip speeds by analyzing the evolution of the three characteristic fractions (Fig. 18). We chose the speed of $400 \mathrm{rpm}$ at the $1.9 \mathrm{~L}$ scale and $460 \mathrm{rpm}$ at the $0.9 \mathrm{~L}$ scale corresponding to impeller tip speeds of 2.93 and $2.89 \mathrm{~m} / \mathrm{s}$, respectively. Fill ratio, binder flow per initial mass of dry powder, chopper speed, homogenization protocol, and granulation time have been kept constant between scales. The wetting stage is similar occurring up until an S/L ratio of $30 \%$ is reached. Afterwards, nucleation and growth start for the small scale, with coarse granules beginning to show at $30 \% \mathrm{~L} / \mathrm{S}$ ratio, while for the larger scale, coarse granules begin to form only from $50 \% \mathrm{~L} / \mathrm{S}$ ratio onwards. This behavior could be explained by the more confined volume of the $0.9 \mathrm{~L}$ bowl causing an increase in contacts between granules, which leads to an acceleration of growth mechanisms.

\section{Conclusion}

The effect of the main means of agitation in a high shear mixer has been investigated in this study. We found that torque curves can allow good control over the granulation process especially at high impeller speeds. At intermediate impeller speeds, torque curves show a low reproducibility especially because of the strong cohesive nature of the employed grade of microcrystalline cellulose. We found that it is important to study the effect of chopper presence at more than just one speed as by increasing impeller speed the percentage of lumps will increase for low to medium impeller speeds and decrease when impeller speed alone can break up the lumps for high impeller speeds. As a general rule, the chopper allows for better binder distribution in the Mi-Pro and is found to be necessary for successful granulation at low to moderate impeller speeds. For high impeller speeds in excess of $4.4 \mathrm{~m} / \mathrm{s}$ with or without chopper, similar granule sizes and growth mechanisms are observed.

Granule roundness was found to increase with impeller speed up to a certain speed, after which granule roundness was found to decrease with increasing impeller speed, most probably because of increased breakage of the granules. Dry granule strength has been found to increase with increasing impeller speed presenting only a slight decrease at the highest impeller speed studied.

The effect of equipment geometry between the 0.9 and the $1.9 \mathrm{~L}$ Mi-pro high shear mixers has shown that at the lower scale, accelerated granule growth takes place while keeping a constant impeller tip speed can give good agreement in terms of size between the scales.

\author{
List of symbols \\ $A^{\prime} \quad$ the area flux of powder traversing the spray zone $\left(\mathrm{m}^{2}\right)$ \\ $A_{g} \quad$ is the measured granule area $\left(\mathrm{m}^{2}\right.$ \\ $C$ material constant (for uniform sphere $C=6$ ) \\ $d_{\mathrm{d}} \quad$ the droplet diameter \\ $d_{\mathrm{i}} \quad$ primary particle diameter $(\mathrm{m})$
}

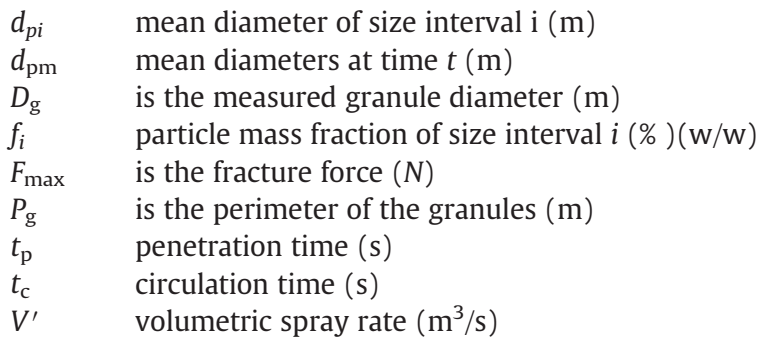

Greek symbols

$\sigma_{\mathrm{g}} \quad$ tensile strength $(\mathrm{Pa})$

$\psi_{\mathrm{a}} \quad$ adimensional spray flux (-)

$\tau_{\mathrm{h}} \quad$ adimensional penetration time $(-)$

\section{References}

[1] M.B. Mackaplow, L.A. Rosen, J.N. Michaels, Effect of primary particle size on granule growth and endpoint determination in high-shear wet granulation, Powder Technol. 108 (2000) 32-45.

[2] G. Betz, P.J. Burgin, H.L. Leuenberger, Power consumption profile analysis and tensile strength measurements during moist agglomeration, Int. J. Pharm. 252 (2003) 11-25.

[3] J.N. Michaels, L. Farber, G.S. Wong, K. Hapgood, S.J. Heidel, J. Farabaugh, J.-H. Chou, G.I. Tardos, Steady states in granulation of pharmaceutical powders with application to scale-up, Powder Technol. 189 (2009) 295-303.

[4] T. Schaefer, B. Taagegard, L.J. Thomsen, H.G. Kristensen, Melt pelletization in a high shear mixer. V. Effects of apparatus variables, Eur. J. Pharm. Sci. (1993) 133-141.

[5] P.C. Knight, An investigation of the kinetics of granulation using a high shear mixer, Powder Technol. 77 (1993) 159-169.

[6] A. Faure, P. York, R. Rowe, Process control and scale-up of pharmaceutical wet granulation processes: a review, Eur. J. Pharm. Biopharm. 52 (2001) 269-277.

[7] K. Saleh, L. Vialatte, P. Guigon, Wet granulation in a batch high shear mixer, Chem. Eng. Sci. 60 (2005) 3763-3775.

[8] M. Benali, V. Gerbaud, M. Hemati, Effect of operating conditions and physicochemical properties on the wet granulation kinetics in high shear mixer, Powder Technol. 190 (2009) 160-169.

[9] A.M. Bouwman, M.R. Visser, G.M.H. Meesters, H.W. Frijlink, The use of Stokes deformation number as a predictive tool for material exchange behaviour of granules in the 'equilibrium phase' in high shear granulation, Int. J. Pharm. 318 (1-2) (2006) 78-85.

[10] H.L. Leuenberger, M. Puchkov, E. Krausbacher, G. Betz, Manufacturing pharmaceutical granules: is the granulation end-point a myth, 3rd Int. Granulation Workshop, , 2007 Sheffield.

[11] H.L. Leuenberger, Granulation: new techniques, Pharm. Acta Helv. 57/3 (1982) $72-82$.

[12] P. Kleinebudde, Shrinking and swelling properties of pellets containing microcrystalline cellulose (MCC) and low substituted hydroxypropylcellulose (L-HPC). I. Shrinking properties, Int. J. Pharm. 104 (1994) 209-219.

[13] P. Kleinebudde, The crystallite-gel-model for microcrystalline cellulose in wet granulation, extrusion and spheronization, Pharm. Res. 14 (1997) 804-809.

[14] M.J. Adams, M.A. Mullier, J.P.K. Seville, Agglomerate strength measurement using a uniaxial confined compression test, Powder Technol. 78 (1994) 5-13.

[15] K. Hapgood, J.D. Litster, S.R. Biggs, T. Howes, Drop penetration into porous powder beds, J. Colloid Interface Sci. 253 (2002) 353-366.

[16] K.P. Hapgood, J.D. Litster, R. Smith, Nucleation regime map for wet granulation, AIChE J. 49 (2) (2003) 350-361.

[17] D. Ameye, E. Keleb, C. Vervaet, J.P. Remon, E. Adams, D.L. Massart, Scaling-up of a lactose wet granulation process in Mi-Pro high shear mixers, Eur. J. Pharm. Sci. 17 (2002) 247-251. 